\title{
Preliminary jitter stability results for the large UV/optical/infrared (LUVOIR) surveyor concept using a non-contact vibration isolation and precision pointing system
}

Lia W. Sacks*a , Carl Blaurock ${ }^{\mathrm{b}}$, Larry D. Dewell ${ }^{\mathrm{c}}$, Kiarash Tajdaran ${ }^{\mathrm{c}}$, Kuo-Chai Liu ${ }^{\mathrm{a}}$, Christine Collins ${ }^{\mathrm{a}}$, Garrett J. West ${ }^{\mathrm{a}}$, Kong Q. Ha ${ }^{\mathrm{a}}$, Matthew R. Bolcar ${ }^{\mathrm{a}}$, Julie A. Crooke ${ }^{\mathrm{a}}$, Jason E. Hylan ${ }^{\mathrm{a}}$, Raymond M. Bell ${ }^{\mathrm{b}}$

${ }^{a}$ NASA Goddard Space Flight Center, 8800 Greenbelt Rd, Greenbelt, MD, USA 20771; ${ }^{\text {N Nightsky }}$

Systems Inc., 110 East Montgomery St, Baltimore, MD 21230; 'ockheed Martin Space Systems

Company, Advanced Technology Center, 3251 Hanover St., Palo Alto, CA, USA 94304

\begin{abstract}
The need for high payload dynamic stability and ultra-stable mechanical systems is an overarching technology need for large space telescopes such as the Large Ultraviolet / Optical / Infrared (LUVOIR) Surveyor concept. The LUVOIR concept includes a 15-meter-diameter segmented-aperture telescope with a suite of serviceable instruments operating over a range of wavelengths between $100 \mathrm{~nm}$ to $2.5 \mathrm{um}$. Wavefront error (WFE) stability of less than 10 picometers RMS of uncorrected system WFE per wavefront control step represents a drastic performance improvement over current space-based telescopes being fielded. Through the utilization of an isolation architecture that involves no mechanical contact between the telescope and the host spacecraft structure, a system design is realized that maximizes the telescope dynamic stability performance without driving stringent technology requirements on spacecraft structure, sensors or actuators. Through analysis of the LUVOIR finite element model and linear optical model, the wavefront error and LineOf-Sight (LOS) jitter performance is discussed in this paper when using the Vibration Isolation and Precision Pointing System (VIPPS) being developed cooperatively with Lockheed Martin in addition to a multi-loop control architecture. The multi-loop control architecture consists of the spacecraft Attitude Control System (ACS), VIPPS, and a Fast Steering Mirror on the instrument. While the baseline attitude control device for LUVOIR is a set of Control Moment Gyroscopes (CMGs), Reaction Wheel Assembly (RWA) disturbance contribution to wavefront error stability and LOS stability are presented to give preliminary results in this paper. CMG disturbance will be explored in further work to be completed.
\end{abstract}

Keywords: LUVOIR, non-contact, vibration isolation, ultra-stable, jitter, wavefront error stability

\section{LUVOIR MISSION CONCEPT AND TELESCOPE STABILITY}

The Large Ultraviolet / Optical / Infrared (LUVOIR) Surveyor is a concept mission for a large multi-wavelength serviceable observatory with an ambitious design and science goals to enable advances across a broad range of astrophysics ${ }^{1}$. The LUVOIR architecture covered in this paper is called Architecture A, and consists of a 15-meterdiameter segmented-aperture telescope along with four serviceable instruments; ECLIPS (Extreme Coronagraph for Living Planetary Systems), HDI (High Definition Imager), LUMOS (LUVOIR Ultraviolet Multi Object Spectrograph), and Pollux, an CNES-led and ESA-consortium contributed instrument concept (6 countries and 12 institutions) for a high-resolution UV spectro-polarimeter. These instruments operate over a range of wavelengths between $100 \mathrm{~nm}$ to 2.5 um. Using these instruments to achieve the science goals imposes stringent requirements on the dynamic stability of the telescope mechanical structure. The first requirement is the Line-of-Sight (LOS) be below 0.3 milli-arcseconds RMS per axis. The second requirement is the wavefront error (WFE) stability needs to be less than 10 picometers RMS of uncorrected system WFE per wavefront control step. Presented in this paper is a set of jitter results to determine if LUVOIR's Architecture A is capable of achieving these requirements. Although there have been updates to the current Architecture A, this model is representative of preliminary analysis.

*lia.sacks@ nasa.gov; phone 1301 286-3596; https://www.nasa.gov

Updated 3/20/14 


\subsection{Telescope Pointing and Control Architecture}

The LUVOIR observatory has two elements, the payload and the spacecraft. The payload includes the Optical Telescope Element and Backplane Support Frame as shown Figure 1. The spacecraft includes the spacecraft bus, the sun shield, and the tower up to the 2 Degrees Of Freedom (DOF) gimbal. These two elements are separated by the Vibration Isolation and Precision Pointing System (VIPPS) at the interface outboard of the gimbal. The VIPPS is described in more detail in Section 1.2.

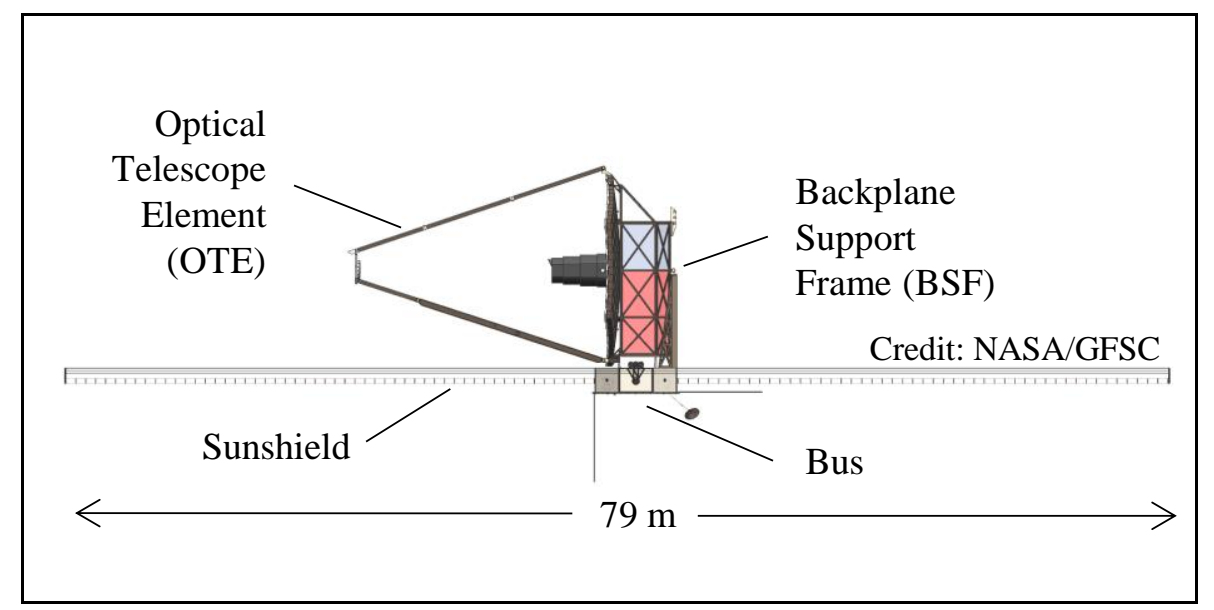

Figure 1: 15-meter LUVOIR Observatory architecture

The control system architecture for the LUVOIR observatory takes advantage of the VIPPS non-contact interface and will use multiple loops to achieve the pointing accuracy and stability required by the mission. As shown in Figure 2, the innermost control loop is on the payload side of the observatory where the Line-of-Sight (LOS) error is estimated through the low order wavefront sensor and/or the High Definition Imager (HDI). This error signal determines the control of the Fast Steering Mirror (FSM). The payload attitude error is determined from the FSM measured angles together with the telescope boresight roll as measured from the payload star tracker measurements. The VIPPS interface non-contact sensors output the payload and spacecraft relative motions and closes both the payload attitude control loop and payload relative control loop around the VIPPS interface non-contact actuators to provide full 6-DOF control of the payload. The relative attitude error of the spacecraft drives control moment gyroscopes (CMGs) commands to keep the spacecraft at a defined separation distance from the payload. Preliminary VIPPS architecture designs, in addition to supporting the steady-state observatory operations, also support observatory agility and repositioning, while maintaining no mechanical contact between the spacecraft and payload; see [7] for analysis of LUVOIR system agility with the VIPPS architecture. 


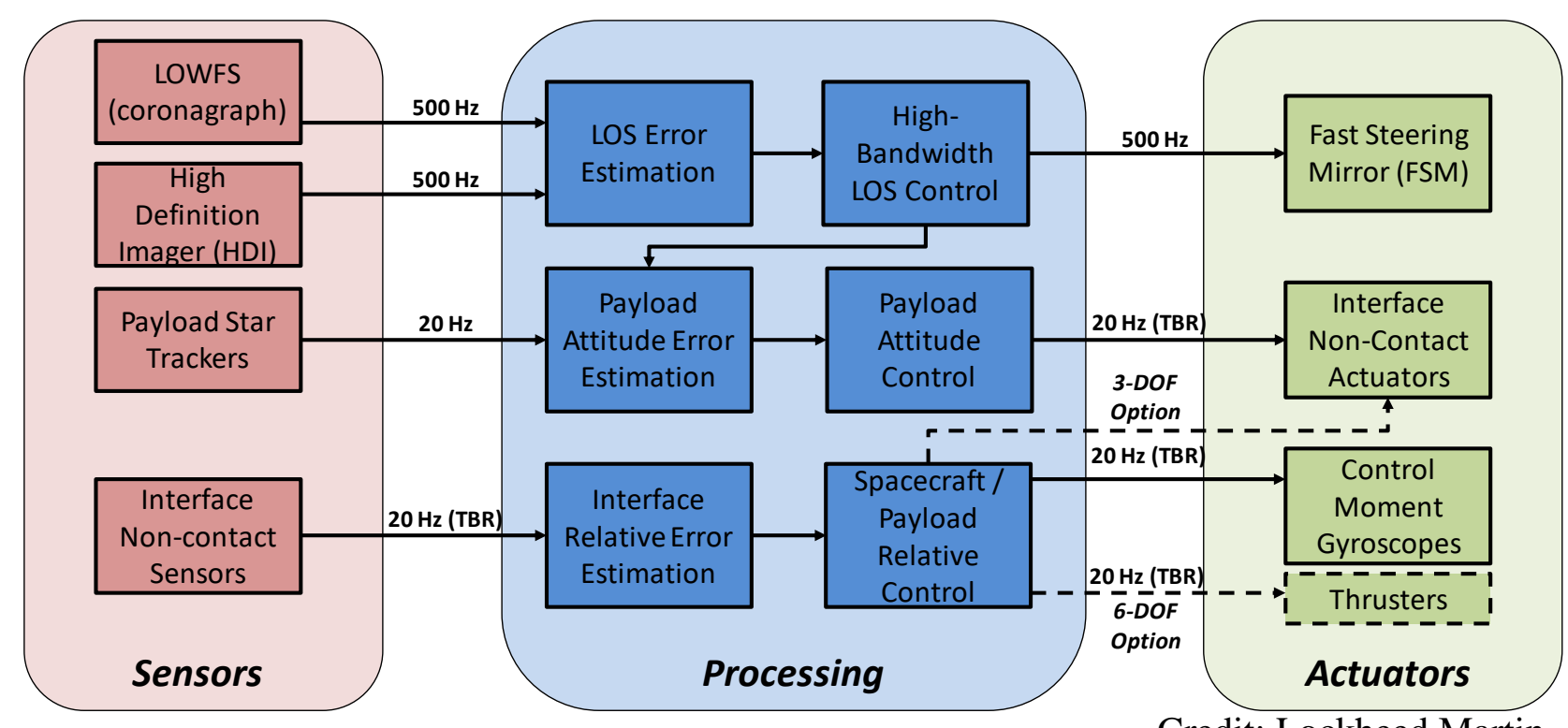

Figure 2: LUVOIR Steady-State Observation Control Architecture

\subsection{Vibration Isolation and Precision Pointing System}

The VIPPS currently being designed for the LUVOIR mission is an isolation architecture that involves no mechanical contact between the telescope and host spacecraft structure. Lockheed Martin has developed and patented a Disturbance Free Payload (DFP) non-contact architecture from which the VIPPS is derived ${ }^{2}$. A DFP-configured observatory is actually two physically-separated bodies flying in close formation, and exchanging forces and torques through noncontact actuators. The payload is controlled by the non-contact actuators and the spacecraft controls its rigid-body degrees of freedom to maintain the desired stroke and gap ${ }^{2}$. Because the disturbances are mainly from the spacecraft and have now been isolated from the payload, requirements on spacecraft structural dynamics and actuator disturbances (arising from reaction wheel or CMG induced vibration, for example) can be similar to current controller designs that exist while still achieving the tight requirements on the observatory. This allows for a wider range of attitude control devices to be considered. Also, the performance of the isolation system is broadband, and isolates the payload from disturbances down to zero frequency. Finally, since the system achieves isolation by virtue of no physical contact, the isolation performance is independent of interface sensor noise characteristics.

\section{LUVOIR INTEGRATED MODEL}

In order to analyze and predict the performance of large telescope systems we can utilize the process of integrated modeling. Integrated modeling consists of creating an overall input-output system model comprised of models from different disciplines. The models used in the LUVOIR integrated model include; structures, optical performance, controls, and disturbances. For structural modeling, a finite element model (FEM) is created to assess the dynamic response of the system being characterized. To model the performance of an optical system like LUVOIR a linear optical model (LOM) can express the optical performance (such as line-of-sight error) as a linear combination of the optical displacement and rotation of specific nodes in the FEM. As a good practice, aligning the nodes in the FEM and those in the LOM created a one-to-one system for mapping disturbances of FEM nodes directly to performance outputs in the LOM. A dynamic model of the VIPPS control system is included to apply forces and torques at the appropriate node locations on the structural model and includes a model of the cables that will cross the VIPPS interface. Last a characterization of the disturbances seen on orbit is added to complete the integrated model. For the analysis presented here only reaction wheel disturbances are introduced; follow-on studies will include detailed modeling of the CMGs that are associated with the baseline LUVOIR architecture. The LUVOIR integrated model utilizes MATLAB and the Nightsky Systems Inc., Disturbance-Optics-Structures-Controls Toolbox to create the end-to-end system. 


\subsection{Finite Element Model}

The FEM, of the LUVOIR observatory was created in Femap Version 11.3.2 and analyses were run using MSC Nastran Version 2016.0.1.

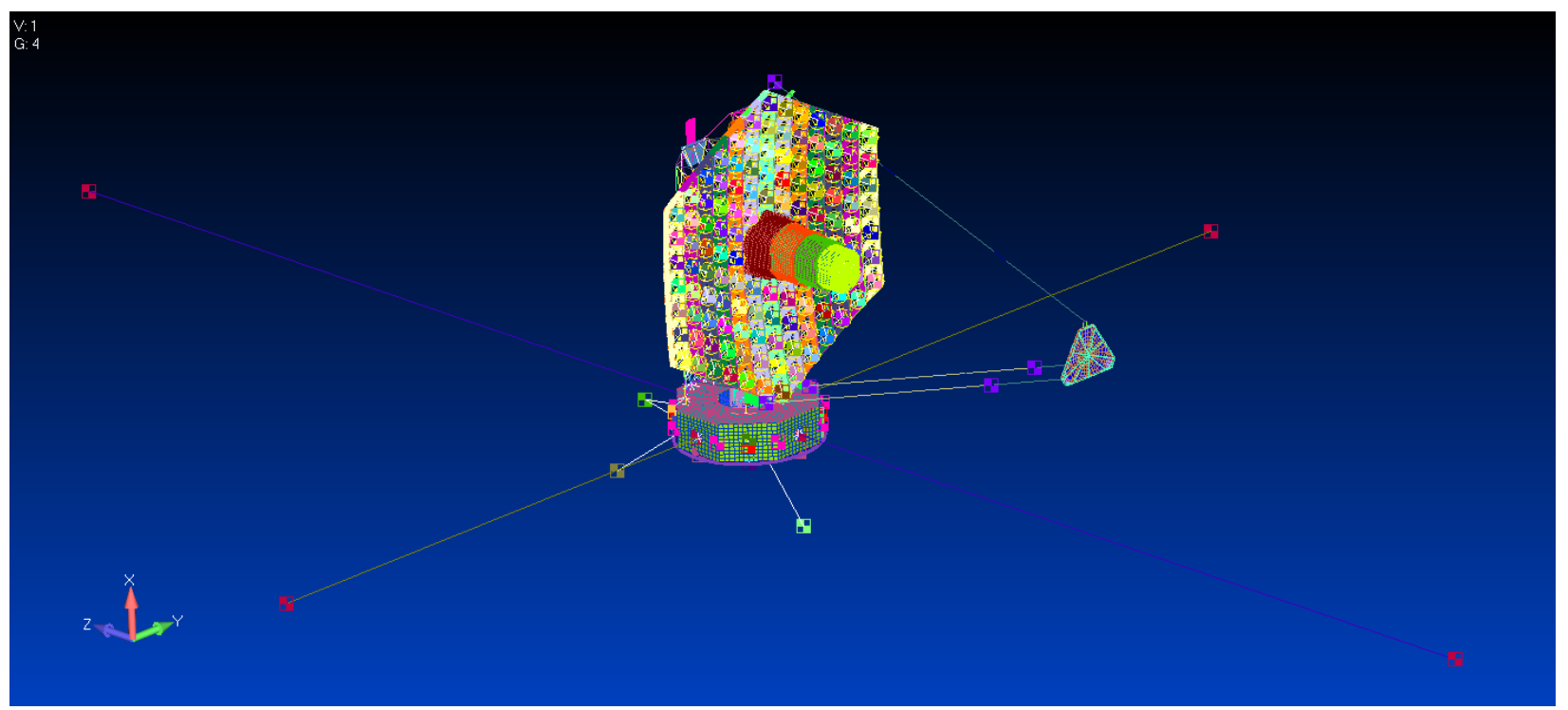

Figure 3: LUVOIR Finite Element Model (FEM)

The LUVOIR FEM is shown in Figure 3. The FEM consisted of 175,000 nodes and 193,000 elements. The structure was modeled using primarily plate and beam elements. Major exceptions to that are the secondary mirror which was constructed using solid elements and each primary mirror segment which was represented using a mass element connected to the mirror backplane using a spring element attached to rigid elements. The mass element and spring element properties were based on inertia and stiffness values from the James Webb Space Telescope primary mirror segment correlated models.

A free dynamics analysis was run on the model for all modes up to $200 \mathrm{~Hz}$, a total of 4373 modes. Displacements were recovered for 131 nodes, of which 125 nodes represented optical components, 4 nodes represented reaction wheels, and 2 nodes represented the isolation system.

The first mode of the observatory was at $0.075 \mathrm{~Hz}$. and was a spacecraft translation along the $\mathrm{Y}$ axis. The first payload mode was mode 19 at $0.53 \mathrm{~Hz}$. and was a rotation about the $\mathrm{X}$ axis.

\subsection{Linear Optical Model}

The LOM consists of a matrix of optical sensitivity data derived from the optical model. The optical model is perturbed in a single degree of freedom and the change in system performance is recorded. The change in performance divided by the perturbation amount is the sensitivity. This process is repeated for every degree of freedom for each mirror in the LUVOIR telescope and the result is a multidimensional matrix of sensitivity data. For the LUVOIR telescope LOM sensitivities are produced for the 6-DOF motion of each mirror, and Surface Figure Errors. For the 6-DOF mirror motions or alignment errors, the change in wavefront and line of sight is recorded. For the surface figure sensitivities, each mirror is distorted using Fringe Zernike Polynomials and the change in wavefront and line of sight is recorded. These sensitivities scale linearly with perturbation amount according to Linear Systems Theory. This simplified optical modelling approach is an efficient way to evaluate the impact of system disturbances such as jitter and thermal drift on the optical performance of the payload. The Matlab code used here to generate the LUVOIR LOM has heritage use from the James Webb Space Telescope (JWST) LOM ${ }^{3,4,5}$.

There are two versions of the LUVOIR LOM: one with a monolithic primary mirror model and the other with a segmented primary mirror. In the monolithic model the primary is modelled as a rigid body. This simplifies the model significantly and allows for easy evaluation of the impact due to primary to secondary mirror misalignment. In the segmented LOM the primary mirror is modelled as 120 separate hexagonal segments. This adds complexity to the model 
but allows for accurate representation of dynamic response of individual segment. The LOM used in this analysis is the monolithic primary mirror model.

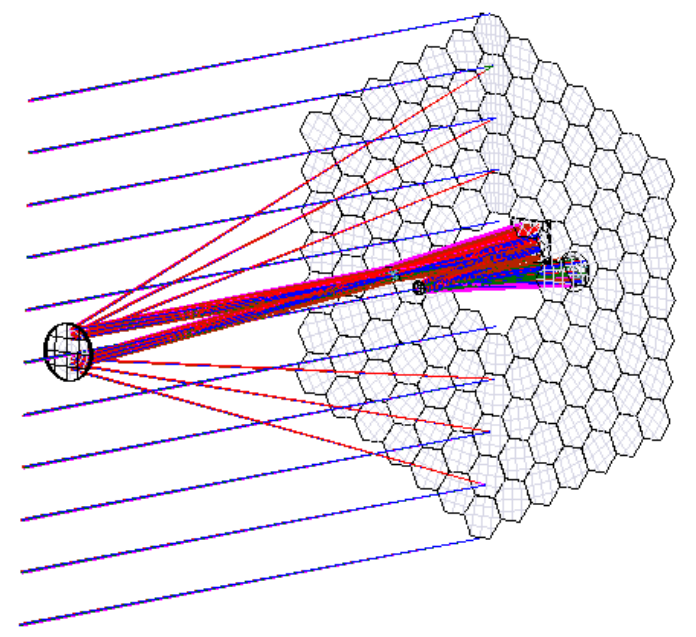

4a)

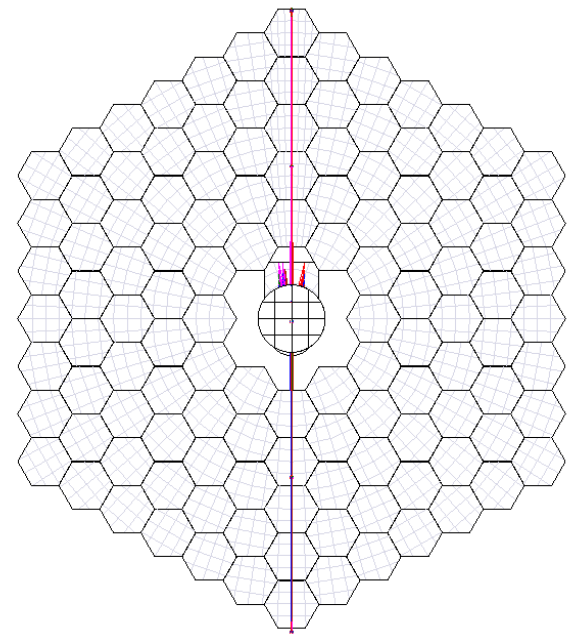

4b)

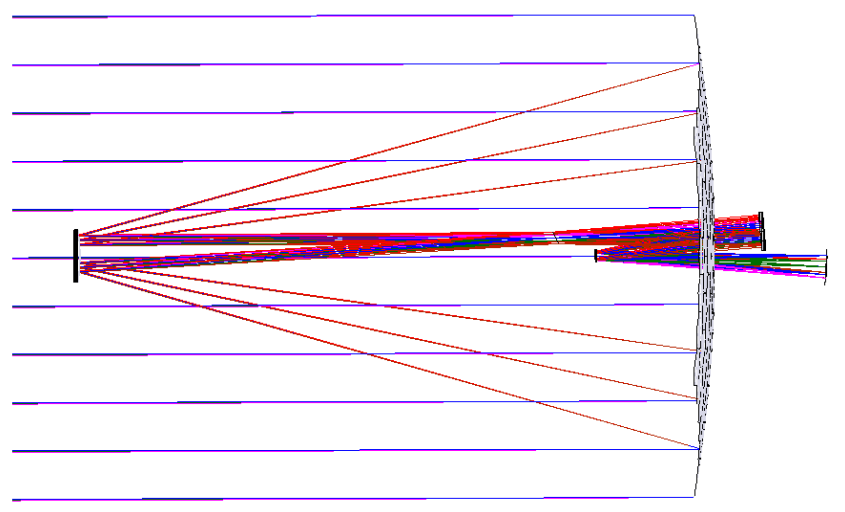

4c)

Figure 4: Linear Optical Model showing the light rays as they reach the primary and secondary mirrors on their way through the optical path. 4a) Off axis view of light rays. 4b) Primary mirror view. 4c) Side view of telescope optical paths.

\subsection{Reaction Wheel Disturbance Model}

For the results presented in this paper the main attitude control devices on the spacecraft are four Honeywell HR14-75 RWAs. The torque capacity of each wheel is $0.14 \mathrm{Nm}$ with a maximum momentum storage of $75 \mathrm{Nms}$. The RWAs are a source of jitter disturbance due to static and dynamic imbalance of the wheels and other bearing imperfections that can introduce disturbances at known ratios of the wheel speed. The RWAs used for this analysis have less capacity than what may be used for the LUVOIR mission and are used as preliminary disturbance inputs until more accurate wheel disturbance models are created.

\subsection{VIPPS Mechanical and Controls Model}

The VIPPS model includes conservative interface cable stiffness and damping based on the JWST. The interface cable stiffness used in this paper is conservative since LUVOIR is estimated to have much fewer physical power and data cables that bridge the spacecraft/payload interface. Also, the interface cable on JWST was assumed to have a temperature of $80 \mathrm{~K}$, but, for LUVOIR, the temperature at the interface is expected to be higher which will result in a lower stiffness. Voice coil actuator coupling was also modeled at the VIPPS interface. The coupling matrix was numerically computed from electro-magnetic finite-element modeling software ${ }^{6}$, for custom large stroke/gap voice coil actuator sized for LUVOIR. 
Baseline VIPPS control parameters were chosen based on a LUVOIR agility study using non-contact vibration isolation ${ }^{7}$. Table 1 summarizes these control parameters. In this preliminary control design, conservative control bandwidths are chosen to avoid interaction between control and structural dynamics.

Table 1. VIPPS Control Parameters

\begin{tabular}{|l|l|}
\hline Controller & Description \\
\hline $\begin{array}{l}\text { Payload inertial } \\
\text { attitude }\end{array}$ & $\begin{array}{l}\text { PID, bandwidth }=0.1 \mathrm{~Hz} ; \text { rate estimator } \\
\text { bandwidth }=0.1 \mathrm{~Hz}\end{array}$ \\
\hline $\begin{array}{l}\text { Interface relative } \\
\text { attitude }\end{array}$ & $\begin{array}{l}\text { PID, bandwidth }=0.01 \mathrm{~Hz} \text {; rate estimator } \\
\text { bandwidth }=50 \mathrm{~Hz}\end{array}$ \\
\hline $\begin{array}{l}\text { Interface relative } \\
\text { translation }\end{array}$ & $\begin{array}{l}\text { PID, bandwidth }=0.06 \mathrm{~Hz} ; \text { rate estimator } \\
\text { bandwidth }=600 \mathrm{~Hz}\end{array}$ \\
\hline
\end{tabular}

\section{INTEGRATED MODEL ANALYSIS AND RESULTS}

Inputting expected RWA disturbance signals at the RWA nodes in the finite element model perturb the system such that the linear optical model sensitivities are used to show the predicted optical performance metrics. With this we have an integrated model that we can iterate on the design to achieve the desired performance outputs. Using the current models and estimated disturbance levels the LOS and Jitter stability results are shown.

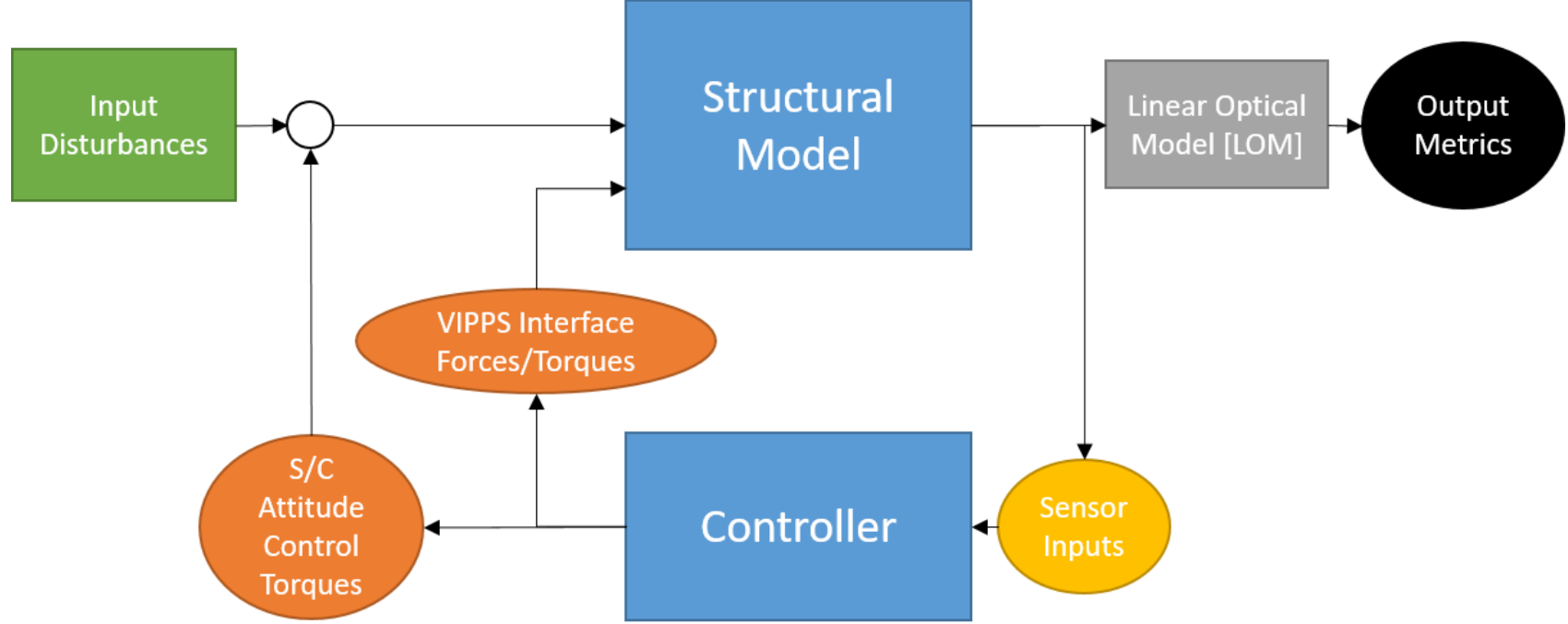

Figure 5: Block diagram of the end-to-end integrated model used for LUVOIR analysis.

In addition to LOS and jitter performance, the integrated model was also used to compute closed-loop isolation transmissibility performance. The transmissibility is a frequency-dependent linear transfer function that relates spacecraft motion to payload motion, in the presence of disturbances applied to the spacecraft center-of-mass. Closedloop linear dynamics for the payload and the spacecraft can be written as follows in the frequency domain:

$$
\left[\begin{array}{l}
\rho_{p}(s) \\
\theta_{p}(s)
\end{array}\right]=\boldsymbol{G}_{c l}^{P}(s)\left[\begin{array}{c}
F_{R W}^{d}(s) \\
T_{R W A}^{d}(s)
\end{array}\right],\left[\begin{array}{c}
\rho_{s}(s) \\
\theta_{s}(s)
\end{array}\right]=\boldsymbol{G}_{c l}^{s}(s)\left[\begin{array}{c}
F_{R W A}^{d}(s) \\
T_{R W A}^{d}(s)
\end{array}\right]
$$

Where $F_{R W A}^{d}$ and $T_{R W A}^{d}$ are the reaction wheel disturbance force and torque vectors, respectively. $\rho_{p}$ and $\rho_{s}$ are the VIPPS node displacement on the payload side and the VIPPS node displacement on the spacecraft side, respectively. $\theta_{p}$ and $\theta_{s}$ are the VIPPS node rotation on the payload side and the VIPPS node rotation on the spacecraft side, respectively. From equation (1), a $6 \times 6$ closed-loop transmissibility matrix can be obtained by eliminating the reaction wheel disturbances: 


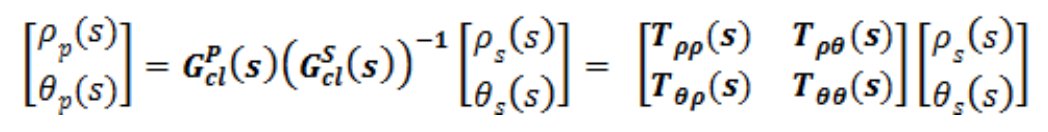

The $6 \times 6$ transmissibility matrix is separated into $43 \times 3$ blocks, as shown in equation (2). In this study, we focus on the maximum singular value magnitude of these 4 matrices.

\subsection{Jitter Performance}

The results from the integrated model analysis show the reaction wheels perturb the system to acceptable levels over all wheel speeds. The two performance metrics measured are the LOS jitter and wavefront error jitter. The motions of the secondary mirror node are expected to contribute to LOS jitter errors and therefore are also shown in Figure 6. Also shown are the motions of the primary mirror node. These show the absolute rigid-body motion of the primary and secondary mirrors relative to the undisturbed node locations. Large motions at low frequency do not contribute to large wavefront error because the primary and secondary mirrors are moving in phase with each other at these frequencies. Because a monolithic primary mirror is used for this analysis, the wavefront error is only a preliminary number, and work is planned to add more realism into the current model which includes a segmented mirror LOM and other sensor and actuator effects.

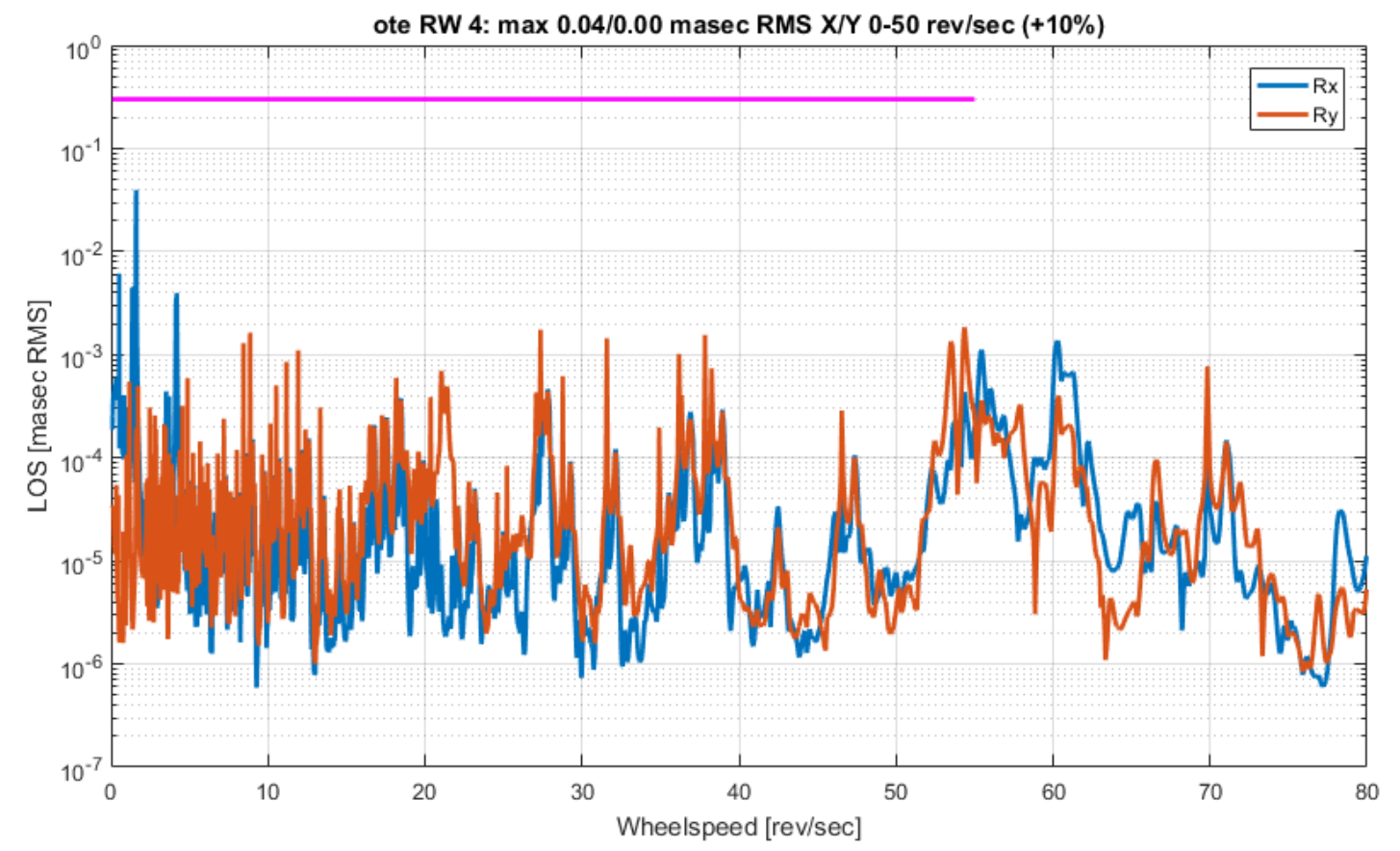

6a) LOS error due to RWA disturbances vs wheel speed against the LUVOIR requirement of 0.3 masec RMS per axis 


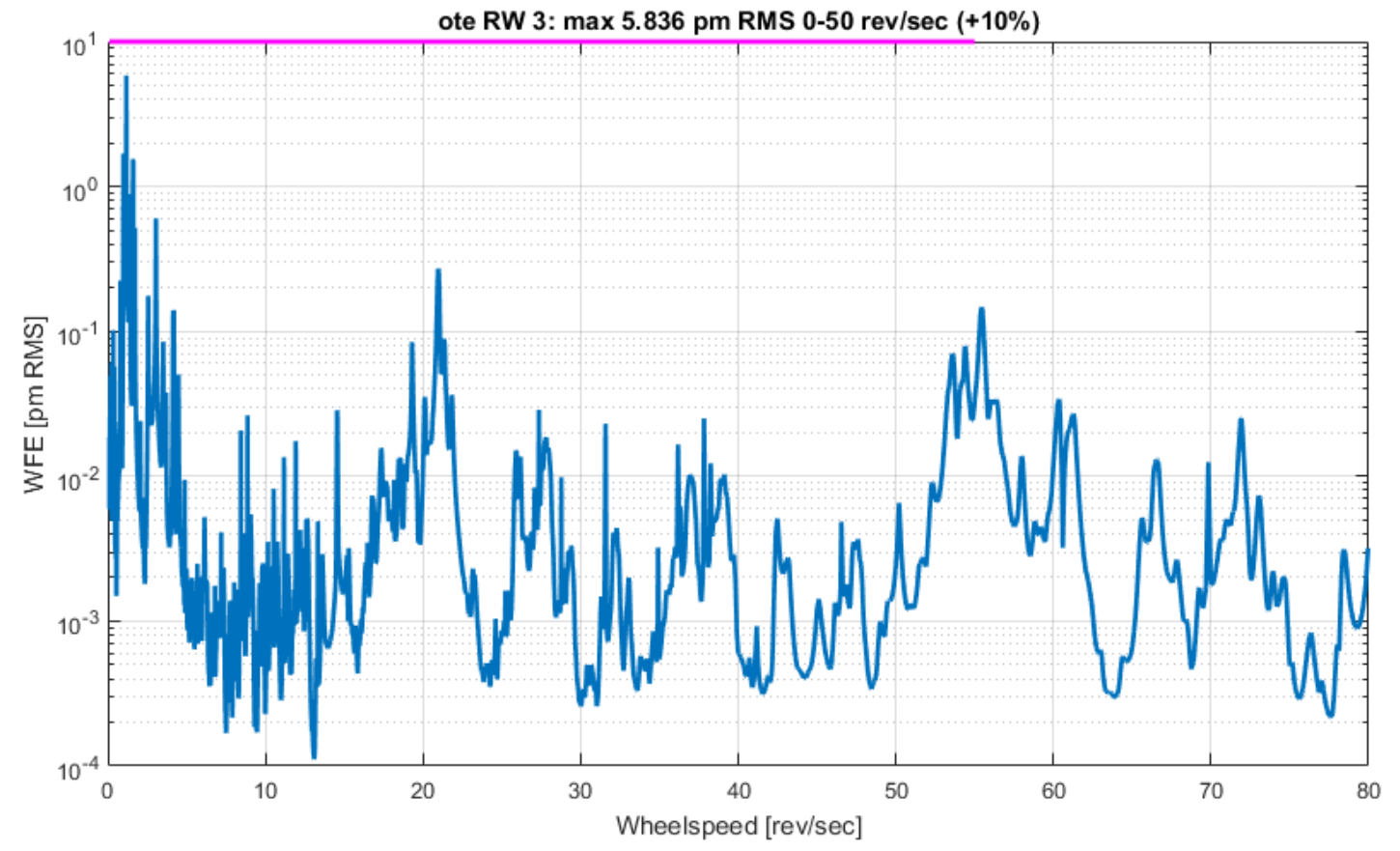

6b) WFE jitter results against the LUVOIR requirement of 10 picometers
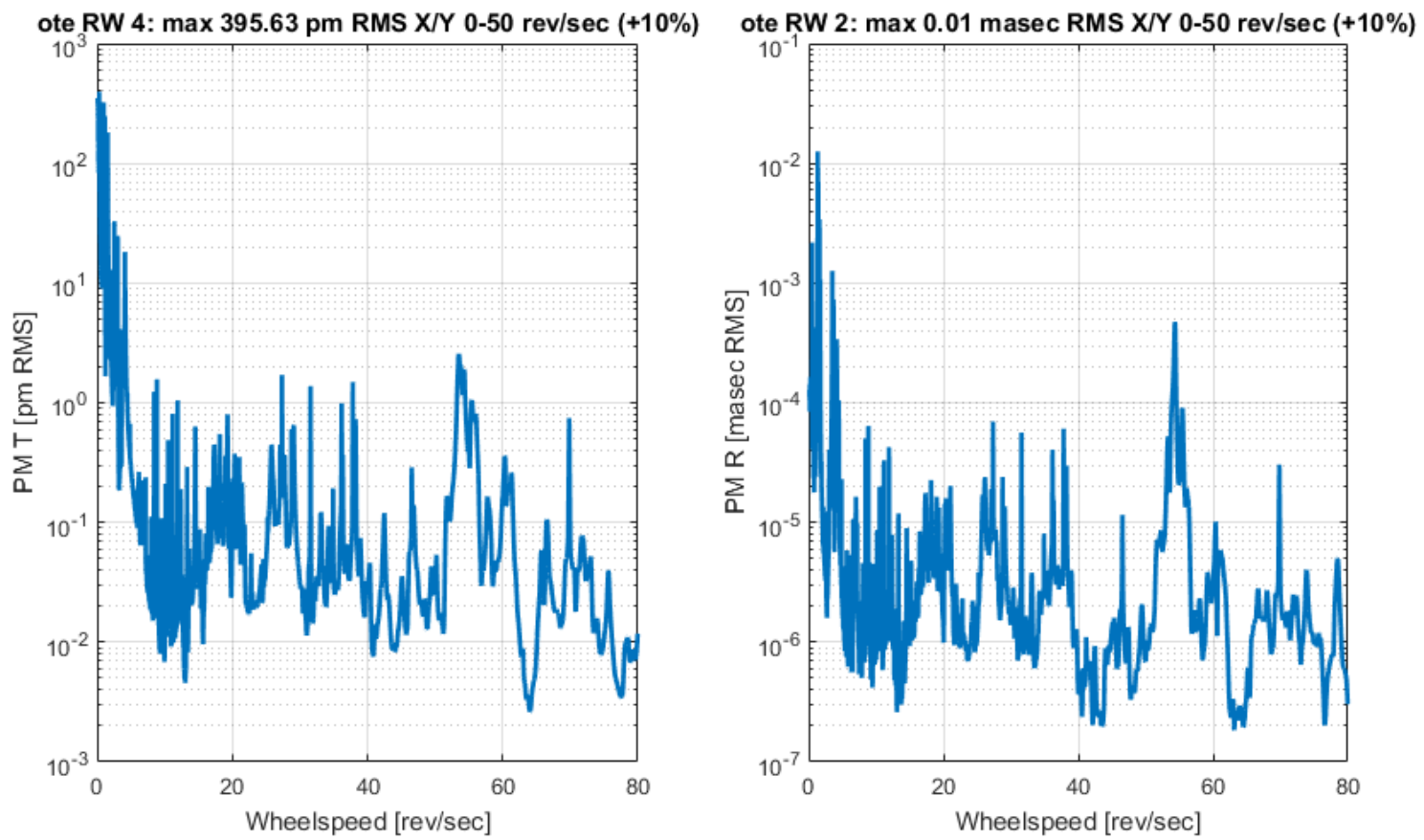

6c) Primary mirror rigid-body absolution motions relative to the undisturbed node location 

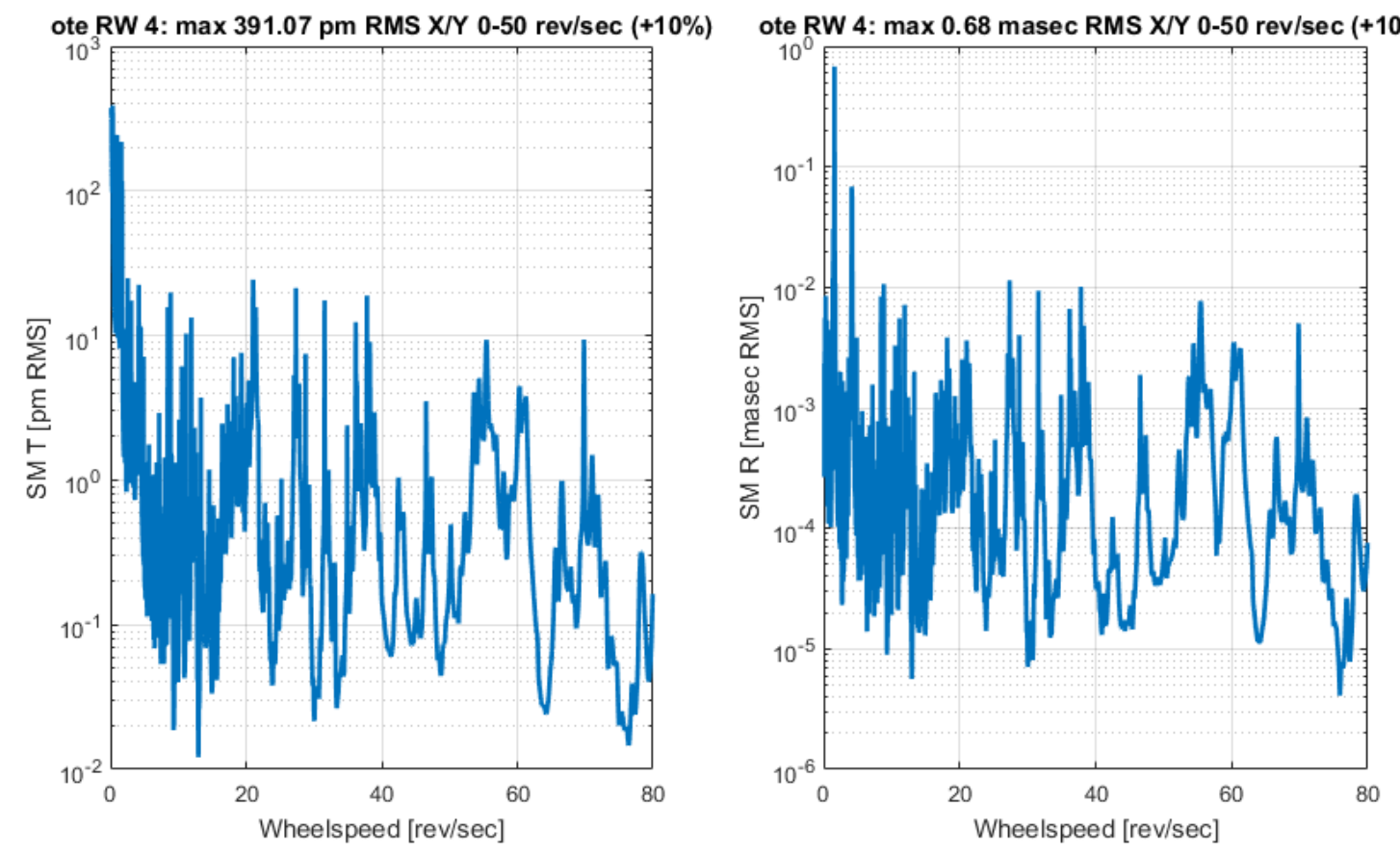

6d) Secondary mirror rigid-body absolute motions relative to the undisturbed node location

\subsection{Closed-loop Transmissibility Performance}

Figure 7 shows the baseline transmissibility performance for LUVOIR. In figure 7a), linear transmissibility shows unity gain at low frequency which is consistent with interface relative control. In figure 7d), the angular transmissibility shows broadband vibration isolation, with a peak angular transmissibility of $-45 \mathrm{~dB}$ and increasing isolation at low frequency. Rigid-body transmissibility performance was also computed based on a rigid-body model for LUVOIR. Comparison between rigid-body transmissibility and flexible-body transmissibility performance shows that the structural resonances in spacecraft and payload can have significant effect on transmissibility results.

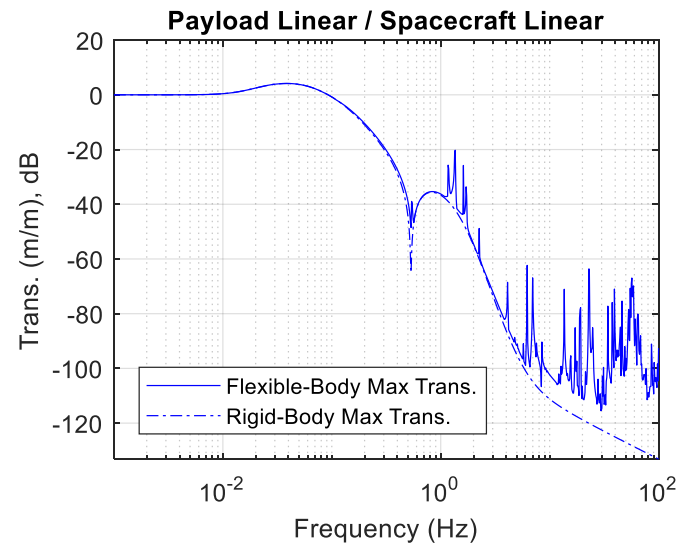

7a)

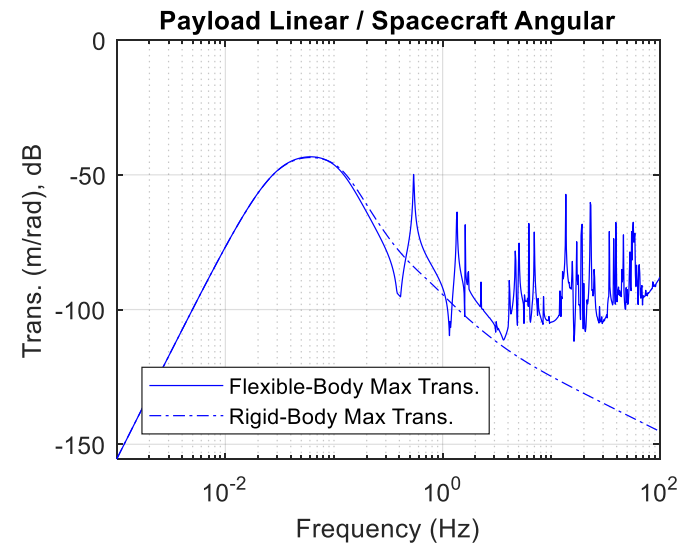

$7 b)$ 


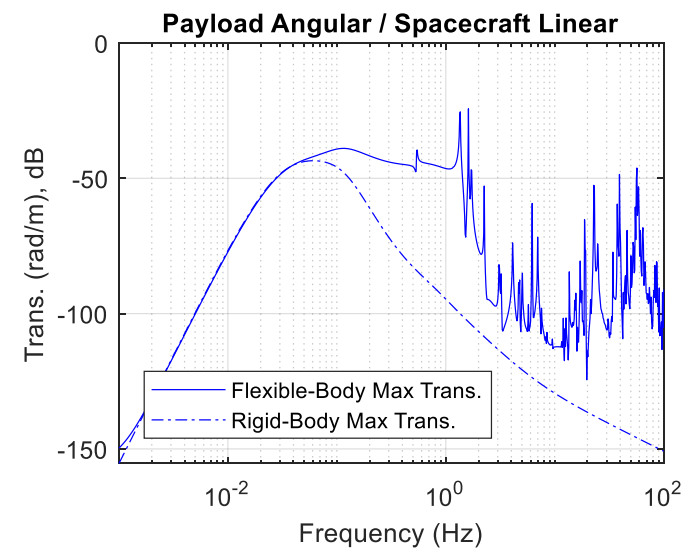

$7 \mathrm{c})$

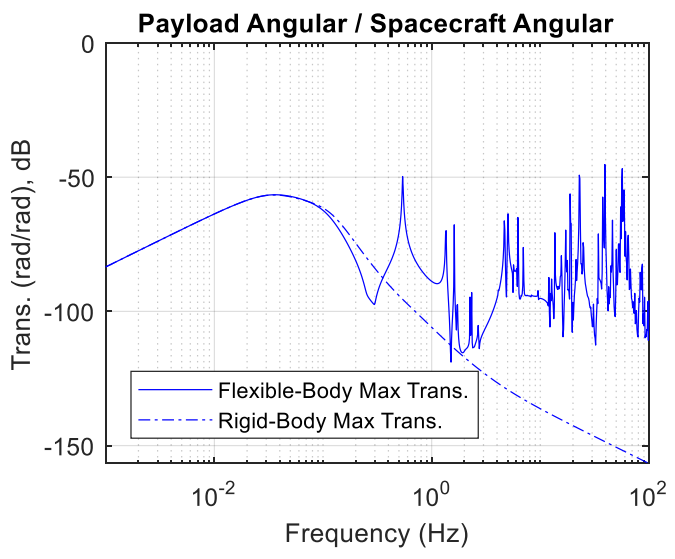

7d)

Figure 7. Closed-loop Transmissibility Performance

\section{CONCLUSION AND FUTURE WORK}

The integrated model connects the structural, optical, and control linear models subject to expected disturbances to determine if the system can meet requirements. Preliminary performance results summarized in this paper, show the isolation performance is sufficient to achieve the tight pointing requirements LUVOIR has put on the system. More analysis and model updates will be carried out (supported in part by NASA's System-Level Segmented Telescope Design program) to further investigate the effects of jitter on the LUVOIR observatory. Future work includes a segmented Linear Optical Model in order to address variations in segment motion which will contribute to the wavefront error in the system, attitude control system design that uses 4 CMGs, and jitter analysis using a CMG disturbance model. Collaboration between NASA and Lockheed Martin was funded under NASA Cooperative Agreement NNG17FH57A.

\section{REFERENCES}

[1] National Aeronautics and Space Administration, Goddard Space Flight Center, "Large UV/Optical/Infrared Surveyor", https://asd.gsfc.nasa.gov/luvoir/.

[2] Dewell, L. et al, "Dynamic Stability with the Disturbance-Free Payload Architecture as Applied to the Large UV/Optical/Infrared (LUVOIR) Mission,” Proc. SPIE 10398 (2017).

[3] Joseph M. Howard, "Optical modeling activities for the James Webb Space Telescope (JWST) project: I. The linear optical model," Proc. SPIE Int. Soc. Opt. Eng. 5178, 82 (2004).

[4] Joseph M. Howard and Kong Ha, "Optical modeling activities for the James Webb Space Telescope (JWST) project: II. Determining image motion and wavefront error over an extended field of view with a segmented optical system," Proc. SPIE 5487, 850 (2004).

[5] Joseph M. Howard, "Optical modeling activities for the James Webb Space Telescope (JWST) project: III. Wavefront Aberrations due to Alignment and Figure Compensation," Proc. SPIE 6675-02 (2007).

[6] ANSYS Maxwell, http://www.ansys.com/products/electronics/ansys-maxwell.

[7] Tajdaran, K. et al, "Telescope line-of-sight slew control and agility with non-contact vibration isolation for the large ultraviolet/optical/infrared (LUVOIR) surveyor concept”, Proc. SPIE 10698-137 (2018). 